(c) 2007 International Press

Adv. Theor. Math. Phys. 11 (2007) 147-174

\title{
On stokes matrices of Calabi-Yau hypersurfaces
}

\author{
Charles F. Doran ${ }^{1}$ and Shinobu Hosono ${ }^{2}$ \\ ${ }^{1}$ Department of Mathematics, University of Washington, Seattle, \\ Washington 98195, USA \\ doran@math.washington.edu \\ ${ }^{2}$ Graduate School of Mathematical Sciences, University of Tokyo, \\ Komaba Meguro-ku, Tokyo 153-8914, Japan \\ hosono@ms.u-tokyo.ac.jp
}

\begin{abstract}
We consider Laplace transforms of the Picard-Fuchs differential equations of Calabi-Yau hypersurfaces and calculate their Stokes matrices. We also introduce two different types of Laplace transforms of Gel'fandKapranov-Zelevinski hypergeometric systems.
\end{abstract}

\section{Introduction - Laplace transforms of period integrals}

Oscillatory integrals play important roles in Landau-Ginzburg theory of an $N=2$ supersymmetric field theory $[1,2]$. They are characterized by certain differential equations with an irregular singularity at infinity, and the Stokes

e-print archive: http://lanl.arXiv.org/abs/math.AG/0608744 
matrices at infinity were key ingredients in the classification program of two dimensional $N=2$ supersymmetric field theories [3] and more recently in the homological mirror symmetry of Fano surfaces $[4,5]$.

In this note, we consider the Landau-Ginzburg theories of Calabi-Yau hypersurfaces in weighted projective spaces and determine the Stokes matrices of the relevant oscillatory integrals. Because of a certain degeneration of the critical values of Landau-Ginzburg potentials, we observe slightly different properties from the Landau-Ginzburg theory of Fano varieties such as the projective spaces $[6,7]$.

Let $X$ be a quintic Calabi-Yau hypersurface in the projective space of dimension 4, and $Y$ be its mirror hypersurface [8]. In the application to mirror symmetry, the mirror hypersurface family appears as a one-parameter family $\left\{Y_{x}\right\}$ of Calabi-Yau hypersurfaces in a toric variety. In the torus $\left(\mathbf{C}^{*}\right)^{4}$, which is dense in the toric variety, we may describe $Y_{x}$ explicitly by first considering $Y_{a}$ as a hypersurface $f(y, a)=0$ with

$$
f(y, a)=y_{1}+y_{2}+y_{3}+y_{4}+\frac{1}{y_{1} y_{2} y_{3} y_{4}}+a .
$$

Since the two hypersurfaces $f(y, a)=0$ and $f(y, \alpha a)=0\left(\alpha^{5}=1\right)$ are isomorphic under $y \rightarrow \alpha y$, we identify $Y_{a}$ with $Y_{\alpha a}$ introducing the parameter $x=-\frac{1}{a^{5}}$ to define the mirror family $\left\{Y_{x}\right\}$.

Now let us take a cycle $\gamma \in H_{3}\left(Y_{x_{0}}, \mathbf{Z}\right)$ and define the following period integral

$$
\Pi_{\gamma}(x)=\frac{1}{(2 \pi i)^{4}} \int_{\gamma} \operatorname{Res}_{f(y, a)=0}\left(\frac{a}{f(y, a)} \prod_{k=1}^{4} \frac{d y_{k}}{y_{k}}\right)
$$

for the family. It is easy to derive the following Picard-Fuchs differential equation,

$$
\left\{\theta_{x}^{4}-5 x\left(5 \theta_{x}+4\right)\left(5 \theta_{x}+3\right)\left(5 \theta_{x}+2\right)\left(5 \theta_{x}+1\right)\right\} \Pi_{\gamma}(x)=0 .
$$

Our interest in this note is the following Laplace transform of this PicardFuchs differential equation,

$$
\left\{z\left(\theta_{z}+1\right)^{3}+5\left(5 \theta_{z}+1\right)\left(5 \theta_{z}+2\right)\left(5 \theta_{z}+3\right)\left(5 \theta_{z}+4\right)\right\} \hat{\Pi}_{\Gamma}(z)=0
$$

where $\hat{\Pi}_{\Gamma}(z)$ is the (formal) Laplace transform of the period integral. When the cycle $\gamma$ is a vanishing cycle, the Laplace transform $\hat{\Pi}_{\Gamma}(z)$ may be written 
more precisely by

$$
\begin{aligned}
\int_{x_{c}}^{\infty} \mathrm{e}^{-z x} \Pi_{\gamma}(x) d x & =\frac{1}{(2 \pi i)^{4}} \int_{x_{c}}^{\infty} \mathrm{e}^{-z x} \int_{\gamma} \operatorname{Res}\left(\frac{1}{W(y)-x} \prod_{k} d y_{k}\right) d x \\
& =\frac{1}{(2 \pi i)^{4}} \int_{\Gamma} \mathrm{e}^{-z W(y)} d y_{1} d y_{2} d y_{3} d y_{4},
\end{aligned}
$$

where we set

$$
W(y)=y_{1} y_{2} y_{3} y_{4}\left(y_{1}+y_{2}+y_{3}+y_{4}+1\right)
$$

and $\Gamma$ represents the Lefschetz thimble made by the vanishing cycle $\gamma$ which vanishes at the critical value $x_{c}$ of $W(y)[9,10] . W(y)$ is called the LandauGinzburg potential, and $\hat{\Pi}_{\Gamma}(z)$ above is the oscillatory integral of the potential. It is easy to see that the Laplace transform (1.4) follows from (1.3) by simple replacements $\theta_{x} \rightarrow-\theta_{z}-1$ and $\frac{\partial}{\partial x} \rightarrow z$ after a division by $x$.

The asymptotics of the oscillatory integral $\hat{\Pi}_{\Gamma}(z)$ when $z \rightarrow \infty$ are determined by the critical values of the potential function $W(y)$. We find that there are two critical values $x_{c}=0$ and $x_{c}=\frac{1}{5^{5}}$ and observe a (continuous) degeneration of the critical points in $W^{-1}(0)$ yet only an isolated critical point in $W^{-1}\left(\frac{1}{5^{5}}\right)$. The degeneration at the critical value 0 is characterized by maximal unipotent monodromy there [11]. In our case, this degeneration results in a certain distinguished property of the solutions about the irregularity at $z=\infty$ of (1.4). The purpose and the main results of this note involve analysis of the asymptotics when $z \rightarrow \infty$ under such a degeneration for the mirror quintic and the similar hypersurfaces studied in [12].

Also, more generally, we introduce two different kinds of Laplace transforms of the Gel'fand-Kapranov-Zelevinski (GKZ) system, $\widehat{\mathrm{GKZ}}^{*}$ and $\widehat{\mathrm{GKZ}}_{\nu}$, and derive the Laplace transform (1.4) from these general systems of differential equations.

This note is organized as follows: In Section 2, we briefly summarize the standard definition of the Stokes lines and then present our analysis of the differential equation (1.4). In Section 3, we will introduce the Laplace transforms $\widehat{\mathrm{GKZ}}^{*}$ and $\widehat{\mathrm{GKZ}}_{\nu}$ of the relevant GKZ system and derive (1.4) from a $\widehat{\mathrm{GKZ}}_{\nu}$ system. Conclusions and discussions are presented in Section 4, where we interpret the Stokes matrices from mirror symmetry. In Appen$\operatorname{dix} \mathrm{A}$, we summarize similar results for other Calabi-Yau hypersurfaces in weighted projective spaces. 


\section{Degeneration and Stokes matrices}

\subsection{Stokes matrices - non-degenerate case}

Here we summarize the description of the asymptotic solutions about an irregular singular point and the definition of Stokes matrices in general. This is to set our notations and also to contrast the general cases with our degenerate cases.

For simplicity, let us consider a linear differential equation of order $n$ which has a regular singularity at $z=0$ and an irregular singularity at $z=\infty$. For example, the differential equation which describes the (small) quantum cohomology of the projective space $\mathbf{P}^{n-1}$ is of this type (see (3.5) below). About $z=0$ we can construct power series solutions of the differential equation with infinite radius of convergence; we denote them by $\phi_{1}(z), \ldots, \phi_{n}(z)$. For the solutions about $z=\infty$, we make the following ansatz for the (formal) solution,

$$
g(z)=\mathrm{e}^{\Lambda(z)} z^{-r} p(z)=\mathrm{e}^{\Lambda(z)} z^{-r}\left(1+\frac{C_{1}}{z}+\frac{C_{2}}{z^{2}}+\cdots\right)
$$

where $\Lambda(z)$ is a polynomial. According to the general theory, by substituting this ansatz into the differential equation, we can determine the polynomial $\Lambda(z)$ and also the constants $r, C_{1}, C_{2}, \ldots$. In this paper, we will call the differential equation non-degenerate if we can find $n$ formal solutions of the above form with linearly independent polynomials $\Lambda_{k}(z)(k=1, \ldots, n)$, and otherwise degenerate.

It is a standard result that even though the series in (2.1) is a formal one, it still provides a way to describe the asymptotics of the holomorphic solutions $\phi_{k}(z)$ near $z=\infty$. Suppose the differential equation is non-degenerate, and write $n$ independent formal solutions $g_{k}(z)=\mathrm{e}^{\Lambda_{k}(x)} z^{-c_{k}} p_{k}(z)(k=1, \ldots, n)$. When $z \rightarrow \infty$, the relative magnitude of $\left|g_{k}(z)\right|$ and $\left|g_{l}(z)\right|$ is governed by the sign of $\operatorname{Re}\left(\Lambda_{k}(z)-\Lambda_{l}(z)\right)$. Namely, $\left|g_{k}(z) / g_{l}(z)\right|>1$ (respectively, $<1$ ) for the asymptotic region defined by $\operatorname{Re}\left(\Lambda_{k}(z)-\Lambda_{l}(z)\right)>0(<0)$. Note that the asymptotic region consists of $d$ connected components, where $d$ is the degree of the polynomial $\Lambda_{k}(z)-\Lambda_{l}(z)$. Each connected component in $|z| \gg 1$ of $\operatorname{Re}\left(\Lambda_{k}(z)-\Lambda_{l}(z)\right)>0$ (respectively $\left.<0\right)$ is called a positive (negative) angular region of $\operatorname{Re}\left(\Lambda_{k}(z)-\Lambda_{l}(z)\right)$. There are $2 d$ angular regions in total.

We will find it convenient, however, to distinguish the angular region written by $\theta<\arg (z)<\theta^{\prime}(|z| \gg 1)$ with its $2 \pi$ rotation, i.e., $\theta+2 \pi<\arg (z)<$ $\theta^{\prime}+2 \pi(|z| \gg 1)$. The Stokes lines are defined to be the half lines from the origin which separate these asymptotic angular regions. 
Since, for the differential equations considered in this note, the polynomial $\Lambda_{k}(z)$ is always linear, we assume hereafter that $\Lambda_{k}(z)=c_{k} z$ with some constant $c_{k}$. In this case, the line $\operatorname{Re}\left(\Lambda_{k}(z)-\Lambda_{l}(z)\right)=0$ simply divides the complex $z$-plane into two connected components, the positive and negative angular regions. We split the line $\operatorname{Re}\left(\Lambda_{k}(z)-\Lambda_{l}(z)\right)=0$ into the two Stokes rays, $R_{k l}$ and $R_{l k}$, so that the counter-clockwise rotation of the half line $R_{i j}$ belongs to the positive angular region of $\operatorname{Re}\left(\Lambda_{i}(z)-\Lambda_{j}(z)\right.$ ) (see figure 1). The notation $\bar{R}_{k l}$ for $R_{l k}$ will be used in the following.

We define

$$
\Xi(\underline{\theta}, \bar{\theta}):=\{z \mid \underline{\theta}<\arg (z)<\bar{\theta}\}
$$

and say that $\Xi(\underline{\theta}, \bar{\theta})$ is a proper angular region of $\Lambda_{k}(z)$ if for each $j \neq k$ there is at most one positive angular region of $\operatorname{Re}\left(\Lambda_{j}(z)-\Lambda_{k}(z)\right)$ which has non-empty intersection with $\Xi(\underline{\theta}, \bar{\theta})$. The following theorem is standard (see e.g., [13]):

Theorem 2.1. Fix the formal solutions $g_{k}(z)(k=1, \ldots, n)$ as above. If $\Xi(\underline{\theta}, \bar{\theta})$ is a proper angular region for all $\Lambda_{k}(z)(k=1, \ldots, n)$, then there are $n$ independent holomorphic solutions $f_{i}(z)(i=1, \ldots, n)$ whose asymptotic expansions in $\Xi(\underline{\theta}, \bar{\theta})$ are given by

$f_{i}(z) \sim g_{i}(z)=e^{\Lambda_{i}(z)} z^{-r_{i}}\left(1+\frac{C_{i 1}}{z}+\frac{C_{i 2}}{z^{2}}+\cdots\right) \quad(z \rightarrow \infty, \quad i=1, \ldots, n)$.

Moreover such solutions $f_{i}(z)$ are unique if $\Xi(\underline{\theta}, \bar{\theta})$ is not contained in any negative angular region $\operatorname{Re}\left(\Lambda_{i}(z)-\Lambda_{j}(z)\right)(1 \leq i, j \leq n)$.

To make use of this theorem, it is convenient to set notation for an angular region on which the solutions $f_{i}(z)$ above are determined uniquely. Let us consider a line $l$ passing through the origin and located in a general position to the Stokes lines. We introduce the orientation of $l$ by writing it as a
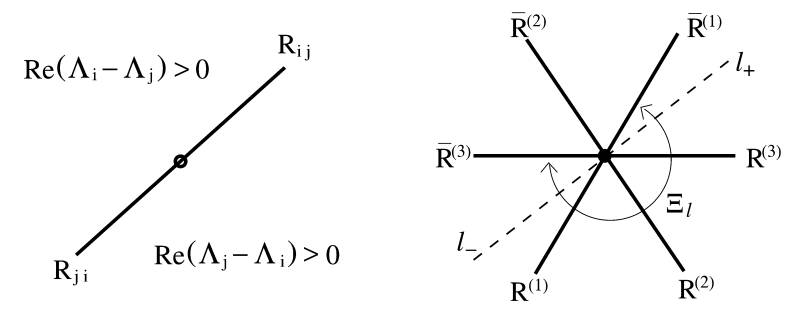

Figure 1: The convention of the Stokes ray $R_{i j}$ explained in the text (left). An example of Stokes lines and the oriented line $l=\left(l_{-}, l_{+}\right)$(right). $\Xi_{l}$ shows the angular region determined by the line $l$. 
union of the half lines from the origin $l_{-}, l_{+}$, i.e., $l=\left(l_{-}, l_{+}\right)$. We define the angular region $\Xi_{l}$ to be the oriented line given by

$$
\Xi_{l}=\Xi\left(\theta_{l_{-}}-\epsilon_{-}, \theta_{l_{+}}+\epsilon_{+}\right)
$$

where $\theta_{l_{-}}$represents $\arg (z)$ for $z \in l_{-}$and $\theta_{l_{+}}=\theta_{l_{-}}+\pi$. The angle $\epsilon_{-}>0$ is determined so that $\theta_{l_{-}}-\epsilon_{-}$represents the angle of the nearest Stokes ray with smaller angle than $l_{-}$. Similarly for $\epsilon_{+}>0$ we use the nearest Stokes ray with larger angle than $l_{+}$. More precisely, $\Xi_{l}$ is defined only up to $2 \pi$ rotations; however, this ambiguity will be fixed in each case when it will become necessary to do so. Also the naive notations $\Xi_{l+\pi}, \Xi_{l-\pi}, \ldots$ are useful if we understand $l+\pi$ (respectively, $l-\pi$ ) to be the counterclockwise (clockwise) rotation by $\pi$ of the oriented line $l=\left(l_{-}, l_{+}\right)$. It is an easy exercise to see that the angular region $\Xi_{l}$ is proper for all $\Lambda_{k}(z)$ and, in addition, is not contained in any negative angular region of $\operatorname{Re}\left(\Lambda_{i}(z)-\right.$ $\left.\Lambda_{j}(z)\right)(1 \leq i, j \leq n)$.

In this note, we adopt the convention of arranging the formal solutions $g_{k}(z)$ and the holomorphic solutions $f_{k}(z)$ into row vectors:

$$
\Psi(z)=\left(g_{1}(z), g_{2}(z), \ldots, g_{n}(z)\right), \quad \Phi(z)=\left(f_{1}(z), f_{2}(z), \ldots, f_{n}(z)\right) .
$$

The following theorem is also standard (see e.g., [13]):

Theorem 2.2. Fix the formal solutions $g_{k}(z)$, and fix the angular region $\Xi_{l}$ with an oriented line $l=\left(l_{-}, l_{+}\right)$. Let $l^{\prime}=\left(l_{-}^{\prime}, l_{+}^{\prime}\right)$ be the oriented line obtained by rotating $l_{-}$in a counter-clockwise direction and passing $l_{-}$through the nearest Stokes ray $R$. Denote the corresponding unique solutions by $\Phi_{l}(z)$ and $\Phi_{l^{\prime}}(z)$, respectively. If the Stokes ray is written $R=R_{i j}$, then these two solutions are related by

$$
\Phi_{l^{\prime}}(z)=\Phi_{l}(z) K_{R_{i j}}, \quad z \in \Xi_{l} \cap \Xi_{l^{\prime}}
$$

with

$$
K_{R_{i j}}=I_{n}+c_{R_{i j}} E_{j i},
$$

where $c_{R_{i j}}$ is a constant, $I_{n}$ is the identity matrix, and $E_{j i}$ is the matrix with non-vanishing entry 1 at the ji position and otherwise 0 . Similarly, let $l^{\prime \prime}$ be the oriented line obtained by clockwise movement of $l_{+}$passing through the nearest Stokes ray $R^{\prime}=R_{j k}$, and $\Phi_{l^{\prime \prime}}(z)$ be the corresponding holomorphic solution on $\Xi_{l^{\prime \prime}}$, then

$$
\Phi_{l^{\prime \prime}}(z)=\Phi_{l}(z) K_{R_{j k}}^{\mathrm{T}}, z \in \Xi_{l} \cap \Xi_{l^{\prime \prime}},
$$

where the superscript $\mathrm{T}$ represents the transpose. 
Note that the constant $c_{R}$ above depends only on the Stokes ray $R$, and also the following relation is implicit in the above theorem;

$$
K_{R_{j k}}^{\mathrm{T}}=K_{R_{k j}}^{-1}\left(=K_{\bar{R}_{j k}}^{-1}\right),
$$

for all Stokes rays. Successive applications of the above theorem give

$$
\begin{aligned}
& \Phi_{l+\pi}(z)=\Phi_{l}(z) K_{R^{(1)}} K_{R^{(2)}} \cdots K_{R^{(n)}} \quad\left(z \in \Xi_{+}\right) \\
& \Phi_{l-\pi}(z)=\Phi_{l}(z) K_{R^{(n)}}^{\mathrm{T}} K_{R^{(n-1)}}^{\mathrm{T}} \cdots K_{R^{(1)}}^{\mathrm{T}} \quad\left(z \in \Xi_{-}\right),
\end{aligned}
$$

where $R^{(1)}, \ldots, R^{(n)}$ are the Stokes rays in the order through which $l_{-}$passes during the rotation by $\pi$ and $\Xi_{ \pm}$are the intersections of the successive angular regions in the process. In the literature, $\Phi_{l}(z), \Phi_{l+\pi}(z)$, and $\Phi_{l-\pi}(z)$ are often denoted by $\Phi^{\text {right }}(z), \Phi^{\text {left }}(z)$ and $\Phi^{\text {left' }}(z)$, respectively, and the above linear relations are written $\Phi^{\text {left }}(z)=\Phi^{\text {right }}(z) S$ and $\Phi^{\text {left' }}(z)=\Phi^{\text {right }}(z) S_{-}$ with

$$
S=K_{R^{(1)}} K_{R^{(2)}} \cdots K_{R^{(n)}}, \quad S_{-}=K_{R^{(n)}}^{\mathrm{T}} K_{R^{(n-1)}}^{\mathrm{T}} \cdots K_{R^{(1)}}^{\mathrm{T}} .
$$

The matrices $S, S_{-}$are the so-called Stokes matrices. By definition, the Stokes matrix $S_{-}$satisfies $S_{-}=S^{\mathrm{T}}$, and the following relation holds:

$$
\Phi^{\text {left }}(z)=\Phi^{\mathrm{left}^{\prime}}\left(\mathrm{e}^{-2 \pi i} z\right) M_{0}, \quad M_{0}=\left(S^{-1}\right)^{\mathrm{T}} S
$$

where $M_{0}$ represents the monodromy about the regular singularity at $z=0$.

\subsection{Stokes matrices - degenerate case}

Now let us turn our attention to our differential equation (1.4) whose irregular singularity is degenerate according to the definition given in section 2.1.

Let us first determine the formal solutions $g_{k}(z)$ near the irregular singularity at $z=\infty$. To describe the solutions, we first define the following hypergeometric series:

$$
G(z, \rho)=\sum_{n=0}^{\infty} \frac{\Gamma(1+5(n+\rho))}{\Gamma(n+\rho+1)^{4}}\left(\frac{1}{z}\right)^{n+\rho+1}
$$

Then it is straightforward to obtain (and define) the following formal solutions. 
Proposition 2.3. The differential equation (1.4) is degenerate, and the following $g_{k}(z)$ are the formal solutions at the irregular singularity $z=\infty$ :

$$
\begin{aligned}
& g_{1}(z)=G(z, 0), \quad g_{2}(z)=\left.\partial_{\tilde{\rho}} G(z, \rho)\right|_{\rho=0}, \quad g_{3}(z)=\left.\left\{\frac{5}{2} \partial_{\tilde{\rho}}^{2}+\mathrm{a} \partial_{\tilde{\rho}}\right\} G(z, \rho)\right|_{\rho=0} \\
& g_{4}(z)=\frac{5^{5} \sqrt{5}}{2 \pi i} e^{-z / 5^{5}} \frac{1}{z^{2}}\left(1-\frac{4375}{z}+\frac{32031250}{z^{2}}-\cdots\right),
\end{aligned}
$$

where $\partial_{\tilde{\rho}}=\frac{1}{2 \pi i} \frac{\partial}{\partial \rho}$ and a is a parameter to be discussed later.

As we see above, $\Lambda_{1}(z)=\Lambda_{2}(z)=\Lambda_{3}(z)=0$ and $\Lambda_{4}(z)=-\frac{z}{5^{5}}$, hence the differential equation is degenerate. The Stokes rays $\bar{R}=R_{4 i}$ and $R=R_{i 4}$ $(i=1,2,3)$ and also the oriented line $l=\left(l_{-}, l_{+}\right)$are defined as before, although their configuration is slightly different (figure 2 ). For the oriented line $l$, we fix the angular region $\Xi_{l}$ by

$$
\Xi_{l}=\Xi\left(-\frac{\pi}{2}, \frac{3 \pi}{2}\right)=\Xi\left(-\frac{3 \pi}{2}, \frac{3 \pi}{2}\right) \cap \Xi\left(-\frac{\pi}{2}, \frac{5 \pi}{2}\right)
$$

and write $\Xi_{l+\pi m}$ for the $\pi m$ rotations of $\Xi_{l}$, i.e., $\Xi_{l+m \pi}=\Xi\left(-\frac{\pi}{2}+m \pi, \frac{3 \pi}{2}+\right.$ $m \pi)$. Note that the angular region $\Xi\left(-\frac{3 \pi}{2}, \frac{3 \pi}{2}\right)$ is proper for $\Lambda_{4}(z)$, and $\Xi\left(-\frac{\pi}{2}, \frac{5 \pi}{2}\right)$ is proper for $\Lambda_{i}(z)(i=1,2,3)$.

The solutions about the regular singularity $z=0$ are given by

$$
\phi_{k}(z)=\sum_{n=0}^{\infty} \frac{\Gamma\left(1+n+\rho_{k}\right)^{3} \Gamma\left(2+n+\rho_{k}\right)}{\Gamma\left(6+5\left(n+\rho_{k}\right)\right)}(-1)^{n} z^{n+\rho_{k}} \text {, }
$$

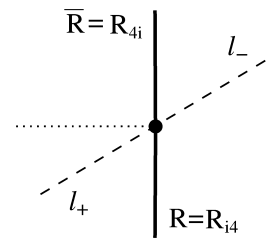

Figure 2: The Stokes rays of (2.3) and the cut of the logarithms (the dotted line) for Calabi-Yau hypersurfaces $Y(d)(d=5,6,8,10)$. 
where $\rho_{k}=-\frac{k}{5}(k=1, \ldots, 4)$. We may write these solutions in terms of Barnes hypergeometric series

$$
\begin{aligned}
& \phi_{1}(z)=\frac{d_{1}}{z^{1 / 5}}{ }_{3} F_{3}\left(\frac{4^{3}}{5} ; \frac{6}{5}, \frac{7}{5}, \frac{8}{5},-\frac{z}{5^{5}}\right), \quad \phi_{2}(z)=\frac{d_{2}}{z^{2 / 5}} F_{3}\left(\frac{3^{3}}{5} ; \frac{4}{5}, \frac{6}{5}, \frac{7}{5},-\frac{z}{5^{5}}\right), \\
& \phi_{3}(z)=\frac{d_{3}}{z^{3 / 5}}{ }_{3} F_{3}\left(\frac{2^{3}}{5} ; \frac{3}{5}, \frac{4}{5}, \frac{6}{5},-\frac{z}{5^{5}}\right), \quad \phi_{4}(z)=\frac{d_{4}}{z^{4 / 5}{ }^{3}} F_{3}\left(\frac{1^{3}}{5} ; \frac{2}{5}, \frac{3}{5}, \frac{4}{5},-\frac{z}{5^{5}}\right),
\end{aligned}
$$

where the constants $d_{1}, d_{2}, d_{3}, d_{4}$ are given, respectively, by $\frac{\Gamma(4 / 5)^{4}}{30}, \frac{\Gamma(3 / 5)^{4}}{10}$, $\frac{\Gamma(2 / 5)^{4}}{5}$, and $\frac{\Gamma(1 / 5)^{4}}{5}$.

To make our definitions parallel to those of the hypergeometric series which appear in [8], we set

$$
\hat{w}_{m}^{\infty}(z)=(1-\alpha) \sum_{k=1}^{4}\left(1-\alpha^{k}\right)^{3} \alpha^{k m+(1 / 2)(k-1)} \phi_{5-k}(z) \quad(m=0,1, \ldots, 4),
$$

with $\alpha=\mathrm{e}^{2 \pi i / 5}$. Note that, by definition, we have

$$
\hat{w}_{0}^{\infty}(z)+\hat{w}_{1}^{\infty}(z)+\cdots+\hat{w}_{4}^{\infty}(z)=0 .
$$

Now we define the holomorphic solutions $f_{k}(z)$ about $z=0$ by

$$
\left(f_{1}(z) f_{2}(z) f_{3}(z) f_{4}(z)\right)=c_{N}\left(\hat{w}_{0}^{\infty}(z) \hat{w}_{1}^{\infty}(z) \hat{w}_{2}^{\infty}(z) \hat{w}_{4}^{\infty}(z)\right) N
$$

where $c_{N}$ is a normalization constant and $N$ is the matrix given in Table 1 . The asymptotics of the solutions $f_{k}(z)$ are given by the following result:

Proposition 2.4. There is a constant $c_{N}$ for which the following asymptotics hold as $z \rightarrow \infty$ :

$$
\begin{aligned}
& f_{k}(z) \sim g_{k}(z)\left(z \in \Xi\left(-\frac{\pi}{2}, \frac{5 \pi}{2}\right), k=1,2,3\right), \\
& f_{4}(z) \sim g_{4}(z)\left(z \in \Xi\left(-\frac{3 \pi}{2}, \frac{3 \pi}{2}\right)\right) .
\end{aligned}
$$

Hence for the angular region $\Xi_{l}=\Xi\left(-\frac{\pi}{2}, \frac{5 \pi}{2}\right) \cap \Xi\left(-\frac{3 \pi}{2}, \frac{3 \pi}{2}\right)$ we have

$$
\Phi_{l}(z)=\left(f_{1}(z) f_{2}(z) f_{3}(z) f_{4}(z)\right) \sim\left(g_{1}(z) g_{2}(z) g_{3}(z) g_{4}(z)\right) \quad(z \rightarrow \infty) .
$$


Table 1: Stokes matrices $K_{\bar{R}}$, the monodromy matrix $L$ of the formal solutions $g_{k}(z)$, and the matrix $N$ in $(2.7)$ for $Y(d)(d=5,6,8,10)$.

$\frac{K}{d}\left(\begin{array}{cccc}1 & 0 & 0 & -5 \\ 0 & 1 & 0 & \mathrm{a}-\frac{5}{2} \\ 0 & 0 & 1 & -1 \\ 0 & 0 & 0 & 1\end{array}\right)$
$\left(\begin{array}{ccccc}1 & 1 & \mathrm{a}+\frac{5}{2} & 0 \\ 0 & 1 & 5 & 0 \\ 0 & 0 & 1 & 0 \\ 0 & 0 & 0 & 1\end{array}\right)\left(\begin{array}{cccc}1 & -\frac{2}{5} & -\frac{2 \mathrm{a}}{5}-2 & 1 \\ 0 & \frac{2}{5} & \frac{2 \mathrm{a}}{5}-2 & -1 \\ 0 & \frac{1}{5} & \frac{\mathrm{a}}{5}-\frac{1}{2} & 0 \\ 0 & -\frac{1}{5} & -\frac{\mathrm{a}}{5}-\frac{1}{2} & 0\end{array}\right)$

$6\left(\begin{array}{cccc}1 & 0 & 0 & -4 \\ 0 & 1 & 0 & \mathrm{a}-\frac{3}{2} \\ 0 & 0 & 1 & -1 \\ 0 & 0 & 0 & 1\end{array}\right)\left(\begin{array}{ccccc}1 & 1 & \mathrm{a}+\frac{3}{2} & 0 \\ 0 & 1 & 3 & 0 \\ 0 & 0 & 1 & 0 \\ 0 & 0 & 0 & 1\end{array}\right)\left(\begin{array}{cccc}1 & -\frac{1}{3} & -\frac{\mathrm{a}}{3}-\frac{3}{2} & 1 \\ 0 & \frac{1}{3} & \frac{\mathrm{a}}{3}-\frac{3}{2} & -1 \\ 0 & \frac{\mathrm{a}}{3}-\frac{1}{2} & 0 \\ 0 & -\frac{1}{3} & -\frac{\mathrm{a}}{3}-\frac{1}{2} & 0\end{array}\right)$

$8\left(\begin{array}{cccc}1 & 0 & 0 & -4 \\ 0 & 1 & 0 & a-1 \\ 0 & 0 & 1 & -1 \\ 0 & 0 & 0 & 1\end{array}\right)\left(\begin{array}{cccc}1 & 1 & a+1 & 0 \\ 0 & 1 & 2 & 0 \\ 0 & 0 & 1 & 0 \\ 0 & 0 & 0 & 1\end{array}\right)\left(\begin{array}{cccc}1 & -\frac{1}{2} & -\frac{a+3}{2} & 1 \\ 0 & \frac{1}{2} & \frac{a-3}{2} & -1 \\ 0 & \frac{1}{2} & \frac{a-1}{2} & 0 \\ 0 & -\frac{1}{2} & -\frac{a+1}{2} & 0\end{array}\right)$

$10\left(\begin{array}{cccc}1 & 0 & 0 & -3 \\ 0 & 1 & 0 & \mathrm{a}-\frac{1}{2} \\ 0 & 0 & 1 & -1 \\ 0 & 0 & 0 & 1\end{array}\right)\left(\begin{array}{cccc}1 & 1 & \mathrm{a}+\frac{1}{2} & 0 \\ 0 & 1 & 1 & 0 \\ 0 & 0 & 1 & 0 \\ 0 & 0 & 0 & 1\end{array}\right)\left(\begin{array}{cccc}1 & 0 & -1 & 1 \\ 0 & 0 & -1 & -1 \\ 0 & 1 & \mathrm{a}-\frac{1}{2} & 0 \\ 0 & -1 & -\mathrm{a}-\frac{1}{2} & 0\end{array}\right)$

The parameter a in each $d$ comes from the definition $g_{3}(z)$ and will be discussed in the final section. 
Our results above rely on numerical calculations of order $10^{-50}$ by Maple. In the numerical calculation, the constant $c_{N}$ is $-0.00342934921 \ldots$

To evaluate the Stokes matrices, we arrange the asymptotic solutions $g_{k}(z)$ as

$$
\Psi(z)=\left(g_{1}(z) g_{2}(z) g_{3}(z) g_{4}(z)\right)
$$

and note that $\Psi(z)$ has the following monodromy due to the logarithms in $g_{k}(z)$ :

$$
\Psi\left(\mathrm{e}^{-2 \pi i} z\right)=\Psi(z) L, \quad L=\left(\begin{array}{cccc}
1 & 1 & \frac{5}{2}+\mathrm{a} & 0 \\
0 & 1 & 5 & 0 \\
0 & 0 & 1 & 0 \\
0 & 0 & 0 & 1
\end{array}\right) .
$$

Now define the holomorphic solutions $\Phi_{l+m \pi}(z)$ about $z=0$ by

$$
\Phi_{l+m \pi}(z)=\left\{\begin{array}{rc}
\left(f_{1}\left(z_{m-1}\right) f_{2}\left(z_{m-1}\right) f_{3}\left(z_{m-1}\right)\right. & \\
\left.f_{4}\left(\mathrm{e}^{-2 \pi i} z_{m-1}\right)\right) L^{-(m-1) / 2} & m: \text { odd } \\
\left(f_{1}\left(z_{m}\right) f_{2}\left(z_{m}\right) f_{3}\left(z_{m}\right) f_{4}\left(z_{m}\right)\right) L^{-m / 2} & m: \text { even }
\end{array}\right.
$$

where we set the notation $z_{s}=\mathrm{e}^{-s \pi i} z$ for integer $s$.

Proposition 2.5. The holomorphic solutions $\Phi_{l+m \pi}(z)(m \in \mathbf{Z})$ have the asymptotics:

$$
\Phi_{l+m \pi}(z) \sim \Psi(z) \quad\left(z \in \Xi_{l+m \pi}, z \rightarrow \infty\right) .
$$

They satisfy the following linear relations

$$
\begin{aligned}
& \Phi_{l+(2 k+1) \pi}(z)=\Phi_{l+2 k \pi}(z) L^{k} K_{\bar{R}} L^{-k}, \\
& \Phi_{l+(2 k+2) \pi}(z)=\Phi_{l+(2 k+1) \pi}(z) L^{k+1} K_{R} L^{-k-1},
\end{aligned}
$$

on the respective angular regions $\Xi_{l+(2 k+1) \pi} \cap \Xi_{l+2 k \pi}$ and $\Xi_{l+(2 k+2) \pi} \cap$ $\Xi_{l+(2 k+1) \pi}$, with the matrices $K_{\bar{R}}, K_{R}$ defined by

$$
K_{\bar{R}}=\left(\begin{array}{cccc}
1 & 0 & 0 & -5 \\
0 & 1 & 0 & -\frac{5}{2}+\mathrm{a} \\
0 & 0 & 1 & -1 \\
0 & 0 & 0 & 1
\end{array}\right), \quad K_{R}=\left(\begin{array}{cccc}
1 & 0 & 0 & 0 \\
0 & 1 & 0 & 0 \\
0 & 0 & 1 & 0 \\
1 & 0 & 0 & 1
\end{array}\right)
$$

Proof. The first half of the claim follows from Proposition 2.4 and the definition of $\Phi_{l+m \pi}(z)$. For example, when $m=2 k+1$, we have for $z \in$ 


$$
\begin{aligned}
& \Xi_{l+(2 k+1) \pi}=\Xi( \\
& \Phi_{l+(2 k+1) \pi}(z)=\left(f_{1}\left(\mathrm{e}^{-2 k \pi i} z\right) f_{2}\left(\mathrm{e}^{-2 k \pi i} z\right) f_{3}\left(\mathrm{e}^{-2 k \pi i} z\right) f_{4}\left(\mathrm{e}^{-(2 k+2) \pi i} z\right)\right) L^{-k} \\
& \\
& \sim\left(g_{1}\left(\mathrm{e}^{-2 k \pi i} z\right) g_{2}\left(\mathrm{e}^{-2 k \pi i} z\right) g_{3}\left(\mathrm{e}^{-2 k \pi i} z\right) g_{4}\left(\mathrm{e}^{-(2 k+2) \pi i} z\right) L^{-k}\right. \\
& =\left(g_{1}(z) g_{2}(z) g_{3}(z) g_{4}(z)\right),
\end{aligned}
$$

where we have used relation (2.8) in the second line, and also $g_{4}\left(\mathrm{e}^{2 \pi i} z\right)=$ $g_{4}(z)$ in the third line. Likewise we can verify the claimed asymptotics for $m=2 k$.

For the second half of the claim, let us note

$$
\phi_{k}\left(\mathrm{e}^{-2 \pi i} z\right)=\alpha \phi_{k}(z) \quad(k=1,2,3,4) .
$$

Then, after some linear algebra using definition (2.7), it is straightforward to verify the following identities,

$$
\begin{aligned}
\left(f_{1}(z) f_{2}(z) f_{3}(z) f_{4}\left(z^{\prime}\right)\right) & =\left(f_{1}(z) f_{2}(z) f_{3}(z) f_{4}(z)\right) K_{\bar{R}}, \\
\left(f_{1}\left(z^{\prime}\right) f_{2}\left(z^{\prime}\right) f_{3}\left(z^{\prime}\right) f_{4}\left(z^{\prime}\right)\right) & =\left(f_{1}(z) f_{2}(z) f_{3}(z) f_{4}\left(z^{\prime}\right)\right) L K_{R},
\end{aligned}
$$

with $z^{\prime}=\mathrm{e}^{-2 \pi i} z$ and the given matrices $K_{\bar{R}}$ and $K_{R}$. These relations are nothing but $\Phi_{l+\pi}(z)=\Phi_{l}(z) K_{\bar{R}}$ and $\Phi_{l+2 \pi}(z) L=\Phi_{l+\pi}(z) L K_{R}$. The general relations (2.10) follow from the above identities applied to $z^{\prime}=\mathrm{e}^{-2 k \pi i} z$, i.e.,

$$
\begin{aligned}
\Phi_{l+(2 k+1) \pi}(z) & =\left(f_{1}\left(z^{\prime}\right) f_{2}\left(z^{\prime}\right) f_{3}\left(z^{\prime}\right) f_{4}\left(\mathrm{e}^{-2 \pi i} z^{\prime}\right)\right) L^{-k} \\
& =\left(f_{1}\left(z^{\prime}\right) f_{2}\left(z^{\prime}\right) f_{3}\left(z^{\prime}\right) f_{4}\left(z^{\prime}\right)\right) K_{\bar{R}} L^{-k}=\Phi_{l+2 k \pi}(z) L^{k} K_{\bar{R}} L^{-k},
\end{aligned}
$$

and a similar formula for the second relation.

Remark 2.6. (1) At first sight, relation (2.10) looks incompatible with the asymptotic behavior (2.9) when we take $z \rightarrow \infty$. However this is not the case due to the triangular nature of the matrices $K_{\bar{R}}, K_{R}, L$ and the asymptotic properties of $g_{k}(z)$.

(2) By relations (2.10), we have $\Phi_{l+\pi}(z)=\Phi_{l}(z) K_{\bar{R}}$ for $z \in \Xi_{l} \cap \Xi_{l+\pi}$ and $\Phi_{l-\pi}(z)=\Phi_{l}(z) K_{R}^{-1}$ for $z \in \Xi_{l} \cap \Xi_{l-\pi}$. From these we read the Stokes matrices as

$$
S=K_{\bar{R}}, \quad S_{-}=K_{R}^{-1} .
$$

Here one should note that relation (2.2), which guarantees the symmetry of exchanging $l_{-}$and $l_{+}$in the argument there, is no longer valid for our degenerate case. Also the Stokes matrices $S$ and $S_{-}$are not constant, but must be conjugated by $L$ in a way that depends on the sheets where the holomorphic solutions $\Phi_{l+m \pi}(z)$ are defined. Of course the latter property simply arises from the logarithmic behavior of the formal series $\Psi(z)=$ $\left(g_{1}(z) g_{2}(z) g_{3}(z) g_{4}(z)\right)$. 


\subsection{Stokes matrices and monodromy matrices}

There are simple relations between the Stokes matrices $K_{R}, K_{\bar{R}}$ and the monodromy matrices of the period integrals $\Pi_{\gamma}(x)$. Let us recall that the Picard-Fuchs differential equation (1.3) has regular singularities at $x=$ $0, \frac{1}{5^{5}}, \infty$, and that the integral symplectic bases of its solutions, first obtained in [8], can be written in a concise way as follows (see $[14,15]$ for some generalizations):

Proposition 2.7. About the regular singularity at $x=0\left(|x|<\frac{1}{5^{5}}\right)$, the integral and symplectic bases of the solutions of the Picard-Fuchs equation (1.3) are given by

$$
\begin{aligned}
& \Pi_{\gamma_{1}}(x)=w(x, 0), \quad \Pi_{\gamma_{3}}(x)=\left.\left\{\frac{5}{2} \partial_{\tilde{\rho}}^{2}+\mathrm{a} \partial_{\tilde{\rho}}\right\} w(x, \rho)\right|_{\rho=0}, \\
& \Pi_{\gamma_{2}}(x)=\left.\partial_{\tilde{\rho}} w(x, \rho)\right|_{\rho=0}, \quad \Pi_{\gamma_{4}}(x)=\left.\left\{-\frac{5}{3 !} \partial_{\tilde{\rho}}^{3}-\frac{50}{12} \partial_{\tilde{\rho}}\right\} w(x, \rho)\right|_{\rho=0},
\end{aligned}
$$

where $w(x, \rho)=\sum_{n=0}^{\infty} \frac{\Gamma(1+5(n+\rho))}{\Gamma(1+n+\rho)^{5}} x^{n+\rho}$. The solutions $\Pi_{\gamma_{k}}(x)$ and $\Pi_{\gamma_{5-k}}(x)$ $(k=1,2)$ are symplectic dual to each other and the constant $\mathbf{a} \in \mathbf{Z}+\frac{1}{2}$ is an arbitrary half (odd) integer.

It is known that the cycle $\gamma_{1} \approx T^{3}$ is a torus cycle coming from the ambient toric variety $[8,16]$. The dual cycle $\gamma_{4}=S^{3}$ is a vanishing cycle that appears at $x=\frac{1}{5^{5}}$ [8]. In our form of the Laplace transform (1.5), we can identify this cycle as the vanishing cycle associated to the critical value $x_{c}=\frac{1}{5^{5}}$ of the LG potential $W(y)$ in (1.6). In fact, after an analytic continuation of $\Pi_{\gamma_{4}}(x)$, we obtain a convergent powerseries in $t=x-\frac{1}{5^{5}}$,

$$
\Pi_{\gamma_{4}}(x)=\frac{5^{5} \sqrt{5}}{2 \pi i}\left(t-\frac{4375}{2} t^{2}+\frac{16015625}{3} t^{3}-\frac{55322265625}{4} t^{4}+\cdots\right),
$$

from which $\Pi_{\gamma_{4}}\left(\frac{1}{5^{5}}\right)=0$ follows (see also Proposition 2.8 below). The cycle $\gamma_{1}$ may also be understood as a "vanishing cycle" which vanishes at a critical point in $W^{-1}(0)$, although in this case the critical points are not isolated in $W^{-1}(0)$. Although the two cycles $\gamma_{1}$ and $\gamma_{4}$ are known explicitly, we lack such explicit description of the cycles $\gamma_{2}$ and $\gamma_{3}$. The above proposition relies only on the requirement that the $\gamma_{k}$ make a symplectic basis. The ambiguity surrounding the description of the cycles $\gamma_{2}$ and $\gamma_{3}$ is the reason for the half integer a is left undetermined. However, from the following Laplace transforms, it is clear that both cycles $\gamma_{2}$ and $\gamma_{3}$ arise from the critical value $x_{c}=0$ of the LG potential $W(y)$ in (1.6). 
Proposition 2.8. Applying Laplace transforms to series (2.11), we obtain the formal series (2.3):

$$
\begin{aligned}
& g_{k}(z)=\int_{0}^{\infty} \mathrm{e}^{-z x} \Pi_{\gamma_{k}}(x) d x \quad(k=1,2,3) \\
& g_{4}(z)=\int_{1 / 5^{5}}^{\infty} \mathrm{e}^{-z x} \Pi_{\gamma_{4}}(x) d x=e^{-z / 5^{5}} \int_{0}^{\infty} e^{-z t} \Pi_{\gamma_{4}}(t) d t .
\end{aligned}
$$

Now let us arrange the period integrals into a row vector

$$
\Pi(x)=\left(\Pi_{\gamma_{1}}(x) \Pi_{\gamma_{2}}(x) \Pi_{\gamma_{3}}(x) \Pi_{\gamma_{4}}(x)\right) .
$$

Then the monodromies about $x=0$ and $x=\frac{1}{5^{5}}$ are represented by the respective matrices acting on this row vector from the right:

$$
M_{0}=\left(\begin{array}{cccc}
1 & 1 & \frac{5}{2}+\mathrm{a} & -5 \\
0 & 1 & 5 & -\frac{5}{2}+\mathrm{a} \\
0 & 0 & 1 & -1 \\
0 & 0 & 0 & 1
\end{array}\right), \quad M_{\mathrm{Con} .}=\left(\begin{array}{cccc}
1 & 0 & 0 & 0 \\
0 & 1 & 0 & 0 \\
0 & 0 & 1 & 0 \\
1 & 0 & 0 & 1
\end{array}\right)
$$

(see [8] for detailed derivations of these results). The following results are now immediate:

Proposition 2.9. The Stokes matrices $K_{R}, K_{\bar{R}}$ in (2.10) satisfy

$$
K_{R}=M_{\text {Con. }}, \quad K_{\bar{R}} L=M_{0} .
$$

The geometric interpretation of these Stokes matrices $K_{R}$ and $K_{\bar{R}}$ is closely related to the (SYZ) geometry [17] which appears at the maximal unipotent monodromy point (maximal degeneracy) and will be discussed in the final section. Here we only remark that the monodromy about $x=\infty$ is represented by $M_{\infty}=M_{\text {Con. }} M_{0}$, and satisfies $M_{\infty}^{5}=1$. Corresponding to these monodromy relations, we have

$$
\begin{aligned}
\Phi_{l+10 \pi}(z) & =\Phi_{l}\left(\mathrm{e}^{-10 \pi i} z\right) K_{\bar{R}} L K_{R} L^{-1}\left(L K_{\bar{R}} L K_{R} L^{-2}\right) \cdots\left(L^{4} K_{\bar{R}} L K_{R} L^{-5}\right) \\
& =\Phi_{l}\left(\mathrm{e}^{-10 \pi i} z\right) K_{\bar{R}} L K_{R} \cdot K_{\bar{R}} L K_{R} \cdots K_{\bar{R}} L K_{R} L^{-5} \\
& =\Phi_{l}\left(\mathrm{e}^{-10 \pi i} z\right) L^{-5}
\end{aligned}
$$

where we use repeatedly the identity

$$
\Phi_{l+(2 k+2) \pi}(z)=\Phi_{l+2 k \pi}\left(\mathrm{e}^{-2 \pi i} z\right) L^{k} K_{\bar{R}} L K_{R} L^{-k-1},
$$

which follows from the definitions. Here, we observe a nice correspondence of each term in the middle line of (2.15) to each movement of the half line 
$l_{-}$passing through the Stokes line and also the cut of the logarithms (see figure 2).

Our analysis presented above extends in a straightforward way to the other Calabi-Yau hypersurfaces studied in [12]. These are given by degree $d$ hypersurfaces $X(d)$ in $\mathbf{P}^{4}(\vec{\omega})=\mathbf{P}^{4}\left(\omega_{1}, \ldots, \omega_{5}\right)$ with their defining data

$$
(d ; \vec{\omega})=\left(5 ; 1^{5}\right),\left(6 ; 2,1^{4}\right),\left(8 ; 4,1^{4}\right),\left(10 ; 5,2,1^{3}\right) .
$$

The mirror Calabi-Yau manifold $Y(d)$ of $X(d)$ is again a hypersurface in a toric variety and the Hodge number $h^{2,1}(Y(d))=1$. In table 1 , we list the Stokes matrix $K_{\bar{R}}$ and also the matrices $L, N$ for each $Y(d)$. The Stokes matrix $K_{R}$ has a common form for all $Y(d)$ (see Proposition 2.5). In Appen$\operatorname{dix} \mathrm{A}$, we present the differential equations, the holomorphic solutions $\Phi_{l}$, and also the asymptotic solutions $g_{k}(z)$ for $d=6,8,10$.

\section{$3 \widehat{\mathrm{GKZ}}^{*}$ versus $\widehat{\mathrm{GKZ}}_{\nu}$ systems}

In Batyrev's formulation of mirror symmetry for hypersurfaces in toric varieties [18], Picard-Fuchs differential equations follow from the more general framework of GKZ hypergeometric systems [16, 19, 20]. In particular, in [20] it was found that GKZ systems in this context are resonant and reducible (and also that we need to consider a certain extension of the GKZ system in general). Here we consider two different types of Laplace transforms of the GKZ system under which the reducibility behaves quite differently.

\subsection{Toric mirror symmetry and the GKZ system}

Let $N \simeq \mathbf{Z}^{4}$ be a lattice and $M \simeq \mathbf{Z}^{4}$ be its dual. An integral polytope in $N \otimes \mathbf{R}$ is called reflexive if it contains the origin as the only interior integral point and all its facets have unit integral distances. Given a reflexive polytope $\Delta$ in $N \otimes \mathbf{R}$, then its polar dual $\Delta^{*}$ in $M \otimes \mathbf{R}$ becomes a reflexive polytope and gives rise to a pair of reflexive polytopes $\left(\Delta, \Delta^{*}\right)$. In the toric mirror duality due to Batyrev, a pair $\left(\Delta, \Delta^{*}\right)$ gives a mirror pair of CalabiYau hypersurfaces $\left(X_{\Delta}, X_{\Delta^{*}}\right)$ in suitable ambient toric varieties [18]. More precisely, the data of a Calabi-Yau hypersurface $X=X_{\Delta}$ gives rise to a family of mirror Calabi-Yau hypersurfaces $Y_{\vec{a}}=Y_{\Delta^{*}}(\vec{a})$ with the defining equation,

$$
f_{\Delta^{*}}(y, \vec{a})=\sum_{\nu \in \Delta^{*} \cap M} a_{\nu} y^{\nu}
$$


where $\vec{a} \in\left(\mathbf{C}^{*}\right)^{\Delta^{*} \cap M}$ denotes the (polynomial) deformations. The period integral we are interested in then takes the form

$$
\Pi(\vec{a})=\frac{1}{(2 \pi i)^{4}} \int_{\gamma} \operatorname{Res}_{f=0}\left(\frac{1}{f_{\Delta^{*}}(y, \vec{a})} \prod_{k=1}^{4} \frac{d y_{k}}{y_{k}}\right),
$$

with a choice of cycle $\gamma \in H_{3}\left(Y_{\vec{a}_{0}}, \mathbf{Z}\right)$. Our expression $\Pi_{\gamma}(x)$ in (1.2) for the mirror quintic follows from this general expression by multiplying with a suitable factor.

Let $A=\left\{(1, \nu) \mid \nu \in \Delta^{*} \cap M\right\}$ and order the elements of $A$ as $\bar{\nu}_{0}, \bar{\nu}_{1}, \ldots, \bar{\nu}_{p}$ $\left(p=\left|\Delta^{*} \cap M\right|-1\right)$ with $\bar{\nu}_{k}=\left(1, \nu_{k}\right)$ and the convention $\bar{\nu}_{0}=(1,0)$ for $0 \in$ $\Delta^{*} \cap M$. Then $\Pi(a)$ satisfies the following $A$-hypergeometric series with exponent $\beta$

$$
\square_{l} \Pi(\vec{a})=0 \quad(l \in L), \quad Z \Pi(\vec{a})=0,
$$

with

$$
\square_{l}=\prod_{l_{i}>0}\left(\frac{\partial}{\partial a_{i}}\right)^{l_{i}}-\prod_{l_{i}<0}\left(\frac{\partial}{\partial a_{i}}\right)^{-l_{i}}, \quad Z=\sum_{i} \bar{\nu}_{i} \theta_{a_{i}}-\beta,
$$

where $a_{i}=a_{\nu_{i}}$ and $L \subset \mathbf{Z}^{p+1}$ is the lattice representing integral relations among the ordered elements in $A$. $Z$ is a differential operator taking its value in $\mathbf{C}^{5}$ and $\beta=(-1,0,0,0,0)$; see [16] for more detailed definitions. We call $A$-hypergeometric systems with the exponent $\beta$ simply GKZ systems. The GKZ system in mirror symmetry is resonant and also reducible in general [20] (see also [21]).

In the following two sections, we consider two different types of Laplace transforms of the GKZ system.

\section{$3.2 \widehat{\mathrm{GKZ}}^{*}$ system}

The set $A$ contains a distinguished point $(1,0)=\left(1, \nu_{0}\right)$ which corresponds to the origin in $\Delta^{*}$. With respect to the variable $a_{0}=a_{\nu_{0}}$, we define the following (formal) Laplace transform:

$$
\hat{\Pi}(z, \bar{a})=\int_{0}^{\infty} \mathrm{e}^{-z a_{0}} \Pi(\vec{a}) d a_{0},
$$

where $\bar{a}=\left(a_{1}, \ldots, a_{p}\right)$. We define the $\widehat{\mathrm{GKZ}}^{*}$ system then as the Laplace transform of the GKZ system, obtained by the following replacements of 
operators in the GKZ system:

$$
\frac{\partial}{\partial a_{0}} \rightarrow z, \quad a_{0} \rightarrow-\frac{\partial}{\partial z}, \quad \theta_{a_{0}} \rightarrow-\theta_{z}-1 .
$$

We note that this system is defined canonically for a given set of toric data of $\Delta^{*}$, since the origin is the unique integral point inside the polytope $\Delta^{*}$. We note further that, in toric geometry, the normal cone at the origin corresponds to the dense orbit $\left(\mathbf{C}^{*}\right)^{4}$ in the toric variety. It is straightforward to see that the Laplace transform (3.2) represents the oscillatory integral of the Landau-Ginzburg theory $\left(f_{\Delta^{*}}(y, \vec{a}),\left(\mathbf{C}^{*}\right)^{4}\right)$ with the Landau-Ginzburg potential $f_{\Delta^{*}}(y, \vec{a})(3.1)$, which was first introduced in [1] and studied further, for example, in [2-4] in exploring the mirror symmetry of Fano varieties.

Example 3.1. As an illustration of the $\widehat{\mathrm{GKZ}}^{*}$ system, let us consider the toric data $\Delta^{*}$ with its integral points given by $\nu_{1}=(1,0,0,0), \nu_{2}=$ $(0,1,0,0), \nu_{3}=(0,0,1,0), \nu_{4}=(0,0,0,1), \nu_{5}=(-1,-1,-1,-1)$, and the origin $\nu_{0}=(0,0,0,0)$. This is the data for the mirror quintic $Y$ and, in fact, the Landau-Ginzburg potential coincides with (1.1). The set $A$ consists of six points $\left(1, \nu_{k}\right)$ in $\mathbf{Z}^{5}$ and has one linear relation which generates the lattice $L$ of rank 1. Under the Laplace transform, the operator $\square_{l}$ corresponding to the generator $l$ of $L$ will be replaced by $\hat{\square}_{l}$ :

$$
\square_{l}=\frac{\partial}{\partial a_{1}} \frac{\partial}{\partial a_{2}} \frac{\partial}{\partial a_{3}} \frac{\partial}{\partial a_{4}} \frac{\partial}{\partial a_{5}}-\left(\frac{\partial}{\partial a_{0}}\right)^{5} \mapsto \hat{\square}_{l}=\frac{\partial}{\partial a_{1}} \frac{\partial}{\partial a_{2}} \frac{\partial}{\partial a_{3}} \frac{\partial}{\partial a_{4}} \frac{\partial}{\partial a_{5}}-z^{5} .
$$

Similarly, the components of the differential operators $Z$ give rise the following operators

$$
-\theta_{z}+\theta_{a_{1}}+\cdots+\theta_{a_{5}}, \quad \theta_{a_{1}}-\theta_{a_{5}}, \quad \theta_{a_{2}}-\theta_{a_{5}}, \quad \theta_{a_{3}}-\theta_{a_{5}}, \quad \theta_{a_{4}}-\theta_{a_{5}},
$$

which annihilate $\hat{\Pi}(z, \bar{a})$. The presence of the latter differential operators implies that $\hat{\Pi}(z, \bar{a})$ is in fact a function of $\tilde{z}=\left(a_{1} a_{2} a_{3} a_{4} a_{5}\right)^{1 / 5} z$, and from $\hat{\square}_{l}$ we finally obtain

$$
\left\{\theta_{\tilde{z}}^{5}-5^{5} \tilde{z}^{5}\right\} \hat{\Pi}(\tilde{z})=0 .
$$

This is the well-studied differential equation of $[6,7]$, where it arose in describing the Frobenius structure of the quantum cohomology of the projective space $\mathbf{P}_{\Delta}=\mathbf{P}^{4}$ with dual polytope $\Delta$.

As we see in the above example, and also observe in general, unlike the original GKZ system, the $\widehat{\mathrm{GKZ}}^{*}$ system is not reducible. This should be contrasted with the second type of Laplace transform below which reproduces the differential equation (1.4) after a factorization of the differential operator. 


\section{$3.3 \widehat{\mathrm{GKZ}}_{\nu}$ system}

Now let us select one variable $a_{\nu}$ for $\nu \neq 0$ and consider the second type of Laplace transform

$$
\Pi(z, \bar{a})=\int_{0}^{\infty} \mathrm{e}^{-z a_{\nu}} \Pi(\vec{a}) d a_{\nu},
$$

where $\bar{a}$ represents the rest of the variables in $\vec{a}$. The corresponding Laplace transform of the GKZ system is obtained by similar replacements to (3.3) involving the variable $a_{\nu}$. We call the resulting set of differential equations the $\widehat{\mathrm{GKZ}}_{\nu}$ system. By contrast with the $\widehat{\mathrm{GKZ}}^{*}$ system, we remark that the $\widehat{\mathrm{GKZ}}_{\nu}$ system depends on the choice of the point $\nu$ up to $G L(4, \mathbf{Z})$ transformations on the polytope $\Delta^{*}$.

Example 3.2. Let us consider the polytope $\Delta^{*}$ of the mirror quintic introduced in Example 3.1, and describe the $\widehat{\mathrm{GKZ}}_{\nu}$ system coming from the choice of the vertex $\nu=\nu_{5}$. In this case, the choice of vertex is unique up to $G L(4, \mathbf{Z})$ transformations. This time the operator $\square_{l}$ for the generator $l$ of $L$ is replaced by the following $\hat{\square}_{l}$ :

$$
\square_{l} \mapsto \hat{\square}_{l}=\frac{\partial}{\partial a_{1}} \frac{\partial}{\partial a_{2}} \frac{\partial}{\partial a_{3}} \frac{\partial}{\partial a_{4}} z-\left(\frac{\partial}{\partial a_{0}}\right)^{5} .
$$

For the components of the operator $Z$, we have

$-\theta_{z}+\theta_{a_{0}}+\cdots+\theta_{a_{4}}, \theta_{a_{1}}+\theta_{z}+1, \theta_{a_{2}}+\theta_{z}+1, \theta_{a_{3}}+\theta_{z}+1, \theta_{a_{4}}+\theta_{z}+1$,

which annihilate $\hat{\Pi}(z, \bar{a})$. These relations imply that $\frac{a_{1} a_{2} a_{3} a_{4}}{a_{0}^{4}} \hat{\Pi}(z, \bar{a})$ is a function of $\tilde{z}=-\frac{a_{0}^{5}}{a_{1} a_{2} a_{3} a_{4}} z$ (where the minus sign is simply a convention). After some manipulations, we obtain the following differential equation from $\hat{\square}_{l}$ :

$$
\left\{\tilde{z} \theta_{a_{1}} \theta_{a_{2}} \theta_{a_{3}} \theta_{a_{4}}+\left(\theta_{a_{0}}-4\right)\left(\theta_{a_{0}}-3\right)\left(\theta_{a_{0}}-2\right)\left(\theta_{a_{0}}-1\right) \theta_{a_{0}}\right\} \hat{\Pi}(z, \bar{a})=0 .
$$

Now define

$$
\hat{\Pi}(\tilde{z})=-\frac{a_{1} a_{2} a_{3} a_{4}}{a_{0}^{4}} \hat{\Pi}(z, \bar{a})
$$

then the differential equation becomes

$$
\theta_{\tilde{z}}\left\{\tilde{z}\left(\theta_{\tilde{z}}+1\right)^{3}+5\left(5 \theta_{\tilde{z}}+1\right)\left(5 \theta_{\tilde{z}}+2\right)\left(5 \theta_{\tilde{z}}+3\right)\left(5 \theta_{\tilde{z}}+4\right)\right\} \hat{\Pi}(\tilde{z})=0 .
$$

Thus we arrive at a reducible differential equation whose irreducible part coincides with our Laplace transform (1.4). It is an easy exercise to derive, 
from (3.6), the following oscillatory integral representation of $\hat{\Pi}(\tilde{z})$,

$$
\hat{\Pi}(\tilde{z})=\frac{1}{(2 \pi i)^{4}} \int_{\Gamma} \mathrm{e}^{-\tilde{z} W(y)} d y_{1} d y_{2} d y_{3} d y_{4},
$$

with the same Laudau-Ginzburg potential as (1.6). For this form of the oscillatory integral, one may infer that the coordinate $\left(y_{1}, y_{2}, y_{3}, y_{4}\right)$ is that of an affine variety. In fact, $y_{1}, \ldots, y_{4}$ may be identified with the coordinate of an affine chart of the non-compact toric variety which is defined by the normal cone of the vertex $\nu=\nu_{5}$. In other words, one may say that (the irreducible part of) the $\widehat{\mathrm{GKZ}}_{\nu}$ system determines the oscillatory integral of the Landau-Ginzburg theory $\left(W(y), \mathbf{C}_{\nu}^{4}\right)$ on the non-compact toric variety $\mathbf{C}_{\nu}^{4}$ determined from the vertex $\nu$. This affine Landau-Ginzburg theory is the one relevant to our (mirror) quintic hypersurface.

In Appendix A, we list the differential equations for the other cases of mirror Calabi-Yau hypersurfaces $Y(d)$. These differential equations also follow from the $\widehat{\mathrm{GKZ}}_{\nu}$ systems under a suitable choice of the vertex $\nu$.

\section{Conclusion and discussions}

By making Laplace transforms of Picard-Fuchs equations of period integrals, we have obtained differential equations with irregular singularities at infinity which are degenerate. We have determined the Stokes matrices of the differential equations. The same differential equations have been obtained by introducing the $\widehat{\mathrm{GKZ}}_{\nu}$ system, a Laplace transform of the GKZ system. We contrasted the $\widehat{\mathrm{GKZ}}_{\nu}$ system with a similar Laplace transform $\widehat{\mathrm{GKZ}}^{*}$, which reproduces the differential equations related to the quantum cohomology of toric varieties.

Although in this note we have restricted our attention to the simplest cases of Calabi-Yau hypersurfaces with one-parameter deformations, similar analyses should be possible for more general Calabi-Yau hypersurfaces starting with our $\widehat{\mathrm{GKZ}}_{\nu}$ system. Also similar Laplace transforms may be considered for Calabi-Yau varieties such as complete intersections in Grassmanians, and in particular for the fourth order differential operators of Calabi-Yau type classified in $[22,23]$. However, in such generalizations, Landau-Ginzburg theories become less clear than for the hypersurface case.

In the rest of this section, we present an interpretation of our Stokes matrices from the perspective of mirror symmetry between $X(d)$ and $Y(d)$. 
In the papers $[8,12]$, the parameter a in the Stokes matrices has been set to $\mathrm{a}=\frac{11}{2}, \frac{9}{2}, 3, \frac{1}{2}$ for $d=5,6,8,10$, respectively. This parameter is directly related to the ambiguity in the quadratic term of the prepotential (the generating function of the Gromov-Witten invariants) and hence plays no role in the study of Gromov-Witten invariants. From our viewpoint of vanishing cycles, however, we should be able to fix this parameter by defining and integrating periods over the symplectic cycles $\gamma_{2}, \gamma_{3}$. Here, from Table 1 , we simply observe that by choosing $\mathrm{a}=\frac{5}{2}, \frac{3}{2}, 1, \frac{1}{2}$ for $d=5,6,8,10$, respectively, the Stokes matrix $K_{\bar{R}}$ simplifies. Furthermore, we may identify the form of the Stokes matrices in terms of the invariants of $X(d)$ as follows:

$$
K_{R}=\left(\begin{array}{cccc}
1 & 0 & 0 & 0 \\
0 & 1 & 0 & 0 \\
0 & 0 & 1 & 0 \\
\chi\left(\mathcal{O}_{X}, \mathcal{O}_{p}\right) & 0 & 0 & 1
\end{array}\right), \quad K_{\bar{R}}=\left(\begin{array}{cccc}
1 & 0 & 0 & -\chi\left(\mathcal{O}_{X}, \mathcal{O}_{X}(1)\right) \\
0 & 1 & 0 & 0 \\
0 & 0 & 1 & -1 \\
0 & 0 & 0 & 1
\end{array}\right)
$$

where $\mathcal{O}_{X}$ and $\mathcal{O}_{p}$ represent the structure sheaf of $X$ and the skyscraper sheaf on a point $p$ of $X$, respectively, and $\mathcal{O}_{X}(1)=\mathcal{O}_{X} \otimes H$, with $H$ the ample line bundle which generates the Picard group $\operatorname{Pic}(X)=\mathbf{Z} H$. Also, for coherent sheaves $\mathcal{E}, \mathcal{F}$ on $X$

$$
\chi(\mathcal{E}, \mathcal{F})=\sum_{k=0}^{3}(-1)^{k} \operatorname{dim} \operatorname{Hom}^{k}(\mathcal{E}, \mathcal{F}) .
$$

By the Riemann-Roch theorem, one may easily verify that $\chi\left(\mathcal{O}_{X}, \mathcal{O}_{X}(1)\right)=$ $5,4,4,3$, for $X(d)(d=5,6,8,10)$, respectively. The fact that the entry -1 in $K_{\bar{R}}$ is common for all $Y(d)$ suggests a similar invariant interpretation, but we shall defer this to future studies. Clearly, these invariant expressions are explained by the homological mirror symmetry conjecture, which implies the correspondence of $\mathcal{O}_{X}$ and $\mathcal{O}_{p}$ with the vanishing cycle $\gamma_{4}=S^{3}$ and the torus cycle $\gamma_{1}=\mathrm{T}^{3}$, respectively $[30,17,24]$. Then, $K_{R}$ represents the Picard-Lefschetz transform for the vanishing cycle $\gamma_{4}$, while $K_{\bar{R}}$ corresponds to the case where the torus cycle $\gamma_{1}$ vanishes. We can see these degenerations at the critical loci in $W^{-1}\left(\frac{1}{5^{5}}\right)$ and $W^{-1}(0)$, respectively, of the the LandauGinzburg potential

$$
W(y)=y_{1} y_{2} y_{3} y_{4}\left(y_{1}+y_{2}+y_{3}+y_{4}+1\right) .
$$

In $W^{-1}\left(\frac{1}{5^{5}}\right)$, we see that the cycle $\gamma_{4} \approx S^{4}$ vanishes and observe the PicardLefschetz transformation in the Stokes matrix $K_{R}$. On the other hand, $W^{-1}(0)$ has a degeneration characterized by maximal unipotent monodromy. Near this degeneration, a torus fibration, called the SYZ fibration [17], has been constructed in $[25,26]$ using the amoeba picture $[27,28]$ of a suitable moment map image of $W^{-1}(\epsilon)$. When $\epsilon$ goes to zero, the moment map 
image $W(y)=\epsilon$ converges to the boundary of a four-dimensional simplex which roughly describes the cycle $\gamma_{4} \approx S^{3}$. The dual cycle $\gamma_{1} \approx T^{3}$ vanishes at each point on the (five) faces of the simplex, which explains the number -5 in $K_{\bar{R}}$. In this picture, the "Picard-Lefschetz" transform $K_{\bar{R}}$ represents a twisting of the zero section $\gamma_{4}$ (cf. [29]). Our missing cycles $\gamma_{2}, \gamma_{3}$ in the symplectic basis should be found in the geometry of $W^{-1}(0)$ in a similar way.

It is worthwhile reproducing here the well-known Stokes matrices $S, S_{-}$of the differential equation (3.5) for the quantum cohomology of the projective space $\mathbf{P}^{4}[6,7]$. Under a suitable choice of basis, the Stokes matrices are calculated in [7] and found to be $S=\left(\chi\left(\mathcal{O}_{\mathbf{P}^{4}}(i), O_{\mathbf{P}^{4}}(j)\right)\right)_{0 \leq i, j \leq 4}^{-1}$ and $S_{-}=$ $S^{\mathrm{T}}$ in terms of the coherent sheaves $\mathcal{O}_{\mathbf{P}^{4}}, \mathcal{O}_{\mathbf{P}^{4}}(1), \ldots, \mathcal{O}_{\mathbf{P}^{4}}(4)$ on $\mathbf{P}^{4}$. In this case, the Landau-Ginzburg potential $f(y, a)$ in (1.1) has five isolated critical points of ordinary double points, and each is considered to be the mirror of $\mathcal{O}_{\mathbf{P}^{4}}(i)$ under a suitable ordering. Thus, we see that although the $\widehat{\mathrm{GKZ}}^{*}$ and $\widehat{\mathrm{GKZ}}_{\nu}$ systems are defined in a similar way starting from the same GKZ system, they have completely different geometric interpretations in mirror symmetry.

\section{A Appendix: Calabi-Yau hypersurfaces in $\mathbf{P}^{4}(\omega)$}

According to [12], we define Calabi-Yau hypersurfaces $X(d)$ in weighted projective spaces $\mathbf{P}^{4}(\vec{\omega})=\mathbf{P}^{4}\left(\omega_{1}, \ldots, \omega_{5}\right)$ for each set of toric data

$$
(d ; \vec{\omega})=\left(5 ; 1^{5}\right),\left(6 ; 2,1^{4}\right),\left(8 ; 4,1^{4}\right),\left(10 ; 5,2,1^{3}\right) .
$$

The mirror $Y(d)$ of $X(d)$ has Hodge number $h^{2,1}(Y(d))=1$. The mirror pairs $(X(d), Y(d))$ are typical examples of Batyrev's mirror symmetry associated to the corresponding pairs of reflexive polytopes $\left(\Delta(\vec{\omega}), \Delta(\vec{\omega})^{*}\right)$. The Laplace transforms of the Picard-Fuchs differential equations listed below follow from the $\widehat{\mathrm{GKZ}}_{\nu}$ under a suitable choice of a vertex $\nu$.

\section{A.1 Series data and Stokes matrices of $Y(d)$}

$Y(5)$ is the quintic mirror hypersurface which has been studied in the text. Since analyses of other cases $Y(d)$ are parallel, we list the corresponding results below, where the items (a) and (b) are the series data near $z=0$ and $z=\infty$, respectively. For convenience, we have included the PicardFuchs equations as well as their Laplace transforms. 
- $Y(6)$ : The Picard-Fuchs equation and its Laplace transform, respectively, are

$$
\begin{aligned}
\left\{\theta_{x}^{4}-9 x\left(6 \theta_{x}+5\right)\left(6 \theta_{x}+4\right)\left(6 \theta_{x}+2\right)\left(6 \theta_{x}+1\right)\right\} \Pi(x) & =0, \\
\left\{z\left(\theta_{z}+1\right)^{3}+9\left(6 \theta_{z}+1\right)\left(6 \theta_{z}+2\right)\left(6 \theta_{z}+4\right)\left(6 \theta_{z}+5\right)\right\} \hat{\Pi}(z) & =0 .
\end{aligned}
$$

(a) We set

$$
\phi_{k}(z)=\sum_{n=0}^{\infty} \frac{\Gamma\left(1+n+\rho_{k}\right)^{3} \Gamma\left(3+2\left(n+\rho_{k}\right)\right)}{\Gamma\left(7+6\left(n+\rho_{k}\right)\right)}(-1)^{n} z^{n+\rho_{k}},
$$

where $\rho_{k}=-\frac{k}{6}$ for $k=1,2,4,5$. We may write these solutions by Barnes hypergeometric series

$$
\begin{array}{ll}
\phi_{1}(z)=\frac{d_{1}}{z^{1 / 6}}{ }_{3} F_{3}\left(\frac{5^{3}}{6} ; \frac{7}{6}, \frac{3}{2}, \frac{5}{3}, \frac{-z}{2^{4} 3^{6}}\right), & \phi_{2}(z)=\frac{d_{2}}{z^{2 / 6}}{ }_{3} F_{3}\left(\frac{2^{3}}{3} ; \frac{5}{6}, \frac{4}{3}, \frac{3}{2}, \frac{-z}{2^{4} 3^{6}}\right), \\
\phi_{4}(z)=\frac{d_{4}}{z^{4 / 6}}{ }_{3} F_{3}\left(\frac{1^{3}}{3} ; \frac{1}{2}, \frac{2}{3}, \frac{7}{6}, \frac{-z}{2^{4} 3^{6}}\right), & \phi_{5}(z)=\frac{d_{5}}{z^{5 / 6}}{ }_{3} F_{3}\left(\frac{1}{6} ; \frac{1}{3}, \frac{1}{2}, \frac{5}{6}, \frac{-z}{2^{4} 3^{6}}\right),
\end{array}
$$

where the constants $d_{1}, d_{2}, d_{4}, d_{5}$ are given by $\frac{\Gamma(2 / 3) \Gamma(5 / 6)}{2^{2} 3^{3}}, \frac{\Gamma(1 / 3) \Gamma(2 / 3)^{3}}{54}$, $\frac{\Gamma(1 / 3)^{3} \Gamma(5 / 3)}{2}$, and $\Gamma\left(\frac{1}{6}\right)^{3} \Gamma(4 / 3)$, respectively. Define

$$
\hat{w}_{m}^{\infty}(z)=(1-\alpha) \sum_{k=1}^{5}\left(1-\alpha^{2 k}\right)\left(1-\alpha^{k}\right)^{2} \alpha^{k m+(1 / 2)(k-1)} \phi_{6-k}(z),
$$

for $0 \leq m \leq 5$ with $\alpha=\mathrm{e}^{2 \pi i / 6}$. Then the holomorphic solution is given by

$$
\Phi_{l}(z)=c_{N}\left(\hat{w}_{0}^{\infty}(z) \hat{w}_{1}^{\infty}(z) \hat{w}_{2}^{\infty}(z) \hat{w}_{5}^{\infty}(z)\right) N,
$$

where $c_{N}=-0.00201572 \ldots$ and the matrix $N$ is given in table 1 .

(b) We set

$$
G(z, \rho)=\sum_{n=0}^{\infty} \frac{\Gamma(1+6(n+\rho))}{\Gamma(1+n+\rho)^{3} \Gamma(1+2(n+\rho))}\left(\frac{1}{z}\right)^{n+\rho+1},
$$

and define the asymptotic solutions

$$
\begin{aligned}
& g_{1}(z)=G(z, 0), g_{2}(z)=\left.\partial_{\tilde{\rho}} G(z, \rho)\right|_{\rho=0}, g_{3}(z)=\left.\left\{\frac{3}{2} \partial_{\tilde{\rho}}^{2}+\mathrm{a} \partial_{\tilde{\rho}}\right\} G(z, \rho)\right|_{\rho=0} \\
& g_{4}(z)=\frac{2^{4} 3^{6} \sqrt{3}}{2 \pi i} \mathrm{e}^{-z /\left(2^{4} 3^{6}\right)} \frac{1}{z^{2}}\left(1-\frac{15876}{z}+\frac{428354568}{z^{2}}-\cdots\right),
\end{aligned}
$$

where $\partial_{\tilde{\rho}}=\frac{1}{2 \pi i} \frac{\partial}{\partial \rho}$ and $\mathrm{a}$ is a parameter. 
- $Y(8)$ : The Picard-Fuchs equation and its Laplace transform, respectively, are

$$
\begin{aligned}
\left\{\theta_{x}^{4}-16 x\left(8 \theta_{x}+7\right)\left(8 \theta_{x}+5\right)\left(8 \theta_{x}+3\right)\left(8 \theta_{x}+1\right)\right\} \Pi(x) & =0, \\
\left\{z\left(\theta_{z}+1\right)^{3}+16\left(8 \theta_{z}+1\right)\left(8 \theta_{z}+3\right)\left(8 \theta_{z}+5\right)\left(8 \theta_{z}+7\right)\right\} \hat{\Pi}(z) & =0 .
\end{aligned}
$$

(a) We set

$$
\phi_{k}(z)=\sum_{n=0}^{\infty} \frac{\Gamma\left(1+n+\rho_{k}\right)^{3} \Gamma\left(5+4\left(n+\rho_{k}\right)\right)}{\Gamma\left(9+8\left(n+\rho_{k}\right)\right)}(-1)^{n} z^{n+\rho_{k}},
$$

where $\rho_{k}=-\frac{k}{8}$ for $k=1,3,5,7$. We may write these solutions by Barnes hypergeometric series

$$
\begin{aligned}
& \phi_{1}(z)=\frac{d_{1}}{z^{1 / 8}}{ }_{3} F_{3}\left(\frac{7^{3}}{8} ; \frac{5}{4}, \frac{6}{4}, \frac{7}{4}, \frac{-z}{2^{16}}\right), \phi_{3}(z)=\frac{d_{3}}{z^{3 / 8}}{ }_{3} F_{3}\left(\frac{5^{3}}{8} ; \frac{3}{4}, \frac{5}{4}, \frac{6}{4}, \frac{-z}{2^{16}}\right), \\
& \phi_{5}(z)=\frac{d_{5}}{z^{5 / 8}}{ }_{3} F_{3}\left(\frac{3^{3}}{8} ; \frac{2}{4}, \frac{3}{4}, \frac{5}{4}, \frac{-z}{2^{16}}\right), \phi_{7}(z)=\frac{d_{7}}{z^{7 / 8}}{ }_{3} F_{3}\left(\frac{1^{3}}{8} ; \frac{1}{4}, \frac{2}{4}, \frac{3}{4}, \frac{-z}{2^{16}}\right),
\end{aligned}
$$

where the constants $d_{1}, d_{3}, d_{5}, d_{7}$ are given by $\frac{\Gamma(1 / 2) \Gamma(7 / 8)^{3}}{2^{8} 3}, \frac{\Gamma(1 / 2) \Gamma(5 / 8)^{3}}{2^{6}}$, $\frac{\Gamma(1 / 2) \Gamma(3 / 8)^{3}}{2^{3}}$, and $\frac{\Gamma(1 / 2) \Gamma(1 / 8)^{3}}{2}$, respectively. Define

$$
\hat{w}_{m}^{\infty}(z)=(1-\alpha) \sum_{k=1}^{7}\left(1-\alpha^{4 k}\right)\left(1-\alpha^{k}\right)^{2} \alpha^{k m+(1 / 2)(k-1)} \phi_{8-k}(z),
$$

for $0 \leq m \leq 7$ with $\alpha=\mathrm{e}^{2 \pi i / 8}$. Then the holomorphic solutions are

$$
\Phi_{l}(z)=c_{N}\left(\hat{w}_{0}^{\infty}(z) \hat{w}_{1}^{\infty}(z) \hat{w}_{2}^{\infty}(z) \hat{w}_{7}^{\infty}(z)\right) N,
$$

where $c_{N}=-0.001316833 \ldots$ and the matrix $N$ is given in table 1 .

(b) We set

$$
G(z, \rho)=\sum_{n=0}^{\infty} \frac{\Gamma(1+8(n+\rho))}{\Gamma(1+n+\rho)^{3} \Gamma(1+4(n+\rho))}\left(\frac{1}{z}\right)^{n+\rho+1}
$$

and define

$$
\begin{aligned}
& g_{1}(z)=G(z, 0), g_{2}(z)=\left.\partial_{\tilde{\rho}} G(z, \rho)\right|_{\rho=0}, g_{3}(z)=\left.\left\{\frac{2}{2} \partial_{\tilde{\rho}}^{2}+\mathrm{a} \partial_{\tilde{\rho}}\right\} G(z, \rho)\right|_{\rho=0}, \\
& g_{4}(z)=\frac{2^{16} \sqrt{2}}{2 \pi i} \mathrm{e}^{-z / 2^{16}} \frac{1}{z^{2}}\left(1-\frac{88064}{z}+\frac{13272875008}{z^{2}}-\cdots\right)
\end{aligned}
$$

where $\partial_{\tilde{\rho}}=\frac{1}{2 \pi i} \frac{\partial}{\partial \rho}$ and $\mathrm{a}$ is a parameter. 
- $Y(10):$ The Picard-Fuchs equation and its Laplace transform, respectively, are

$$
\begin{array}{r}
\left\{\theta_{x}^{4}-2^{4} 5 x\left(10 \theta_{x}+9\right)\left(10 \theta_{x}+7\right)\left(10 \theta_{x}+3\right)\left(10 \theta_{x}+1\right)\right\} \Pi(x)=0 \\
\left\{z\left(\theta_{z}+1\right)^{3}+2^{4} 5\left(10 \theta_{z}+1\right)\left(10 \theta_{z}+3\right)\left(10 \theta_{z}+7\right)\left(10 \theta_{z}+9\right)\right\} \hat{\Pi}(z)=0 .
\end{array}
$$

(a) We set

$\phi_{k}(z)=\sum_{n=0}^{\infty} \frac{\Gamma\left(1+n+\rho_{k}\right)^{2} \Gamma\left(3+2\left(n+\rho_{k}\right)\right) \Gamma\left(6+5\left(n+\rho_{k}\right)\right)}{\Gamma\left(11+10\left(n+\rho_{k}\right)\right)}(-1)^{n} z^{n+\rho_{k}}$,

where $\rho_{k}=-\frac{k}{10}$ for $k=1,3,7,9$. We may write these solutions by Barnes hypergeometric series

$$
\begin{aligned}
& \phi_{1}(z)=\frac{d_{1}}{z^{1 / 10}}{ }_{3} F_{3}\left(\frac{9^{3}}{10} ; \frac{5}{6}, \frac{8}{5}, \frac{9}{5}, \frac{-z}{2^{8} 5^{5}}\right), \\
& \phi_{3}(z)=\frac{d_{3}}{z^{3 / 10}} 3 F_{3}\left(\frac{7^{3}}{10} ; \frac{4}{5}, \frac{7}{5}, \frac{8}{5}, \frac{-z}{2^{8} 5^{5}}\right), \\
& \phi_{7}(z)=\frac{d_{7}}{z^{7 / 10}} 3 F_{3}\left(\frac{3}{10}^{3} ; \frac{2}{5}, \frac{3}{5}, \frac{6}{5}, \frac{-z}{2^{8} 5^{5}}\right), \\
& \phi_{9}(z)=\frac{d_{9}}{z^{9 / 10}} 3 F_{3}\left(\frac{1^{3}}{10} ; \frac{1}{5}, \frac{2}{5}, \frac{4}{5}, \frac{-z}{2^{8} 5^{5}}\right),
\end{aligned}
$$

where the constants $d_{1}, d_{3}, d_{7}, d_{9}$ are given by $\frac{\Gamma(9 / 10)^{2} \Gamma(4 / 5) \Gamma(1 / 2)}{2^{10} 15}$, $\frac{\Gamma(7 / 10)^{2} \Gamma(2 / 5) \Gamma(1 / 2)}{2^{7} 15}, \frac{\Gamma(3 / 10)^{2} \Gamma(3 / 5) \Gamma(1 / 2)}{2^{3}}$, and $\frac{\Gamma(1 / 10)^{2} \Gamma(1 / 5) \Gamma(1 / 2)}{2}$, respectively. Define

$$
\hat{w}_{m}^{\infty}(z)=(1-\alpha) \sum_{k=1}^{9}\left(1-\alpha^{5 k}\right)\left(1-\alpha^{2 k}\right)\left(1-\alpha^{k}\right) \alpha^{k m+(1 / 2)(k-1)} \phi_{10-k}(z),
$$

for $0 \leq m \leq 9$ with $\alpha=\mathrm{e}^{2 \pi i / 10}$. Then the holomorphic solutions are given by

$$
\Phi_{l}(z)=c_{N}\left(\hat{w}_{0}^{\infty}(z) \hat{w}_{1}^{\infty}(z) \hat{w}_{2}^{\infty}(z) \hat{w}_{9}^{\infty}(z)\right) N
$$

where $c_{N}=-0.0001304601 \ldots$ and the matrix $N$ is given in table 1 . 
(b) We set

$$
G(z, \rho)=\sum_{n=0}^{\infty} \frac{\Gamma(1+10(n+\rho))}{\Gamma(1+n+\rho)^{2} \Gamma(1+2(n+\rho)) \Gamma(1+5(n+\rho))}\left(\frac{1}{z}\right)^{n+\rho+1}
$$

and define the asymptotic solutions

$$
\begin{aligned}
& g_{1}(z)=G(z, 0), g_{2}(z)=\left.\partial_{\tilde{\rho}} G(z, \rho)\right|_{\rho=0}, g_{3}(z)=\left.\left\{\frac{1}{2} \partial_{\tilde{\rho}}^{2}+\mathrm{a} \partial_{\tilde{\rho}}\right\} G(z, \rho)\right|_{\rho=0}, \\
& g_{4}(z)=\frac{2^{8} 5^{5}}{2 \pi i} \mathrm{e}^{-z / 2^{8} 5^{5}} \frac{1}{z^{2}}\left(1-\frac{1040000}{z}+\frac{1884800000000}{z^{2}}-\cdots\right)
\end{aligned}
$$

where $\partial_{\tilde{\rho}}=\frac{1}{2 \pi i} \frac{\partial}{\partial \rho}$ and a is a parameter.

\section{A.2 Stokes matrices and monodromy matrices}

As in Proposition 2.8, there are relations between the formal solutions $g_{k}(z)$ and period integrals $\Pi_{\gamma_{k}}(x)$ for a symplectic basis $\gamma_{1}, \ldots, \gamma_{4}$ of $H_{3}(Y(d), \mathbf{Z})$, with $\left|\gamma_{1} \cap \gamma_{4}\right|=\left|\gamma_{2} \cap \gamma_{3}\right|=1$ and vanishing intersections for other pairings. Similar to the case $Y(5)$, we know the cycles $\gamma_{1} \approx T^{3}$ and $\gamma_{4} \approx S^{3}$ explicitly. However, as for $\gamma_{2}$ and $\gamma_{3}$, we do not have precise forms of the cycles. Here we only verify the expected symplectic and integral monodromy of the hypergeometric series $\Pi_{\gamma_{k}}(x)$ defined below $[12,15,22]$.

To define $\Pi_{\gamma_{k}}(x)$ in general, let us set

$$
w(x, \rho)=\sum_{n=0}^{\infty} \frac{\Gamma(1+d(n+\rho))}{\prod_{i=1}^{5} \Gamma\left(1+\omega_{i}(n+\rho)\right)} x^{n+\rho},
$$

for each $Y(d)$ with toric data $(d ; \vec{\omega})$. Then the symplectic bases given in Proposition 2.7 generalize to $Y(d)$ by

$$
\begin{aligned}
& \Pi_{\gamma_{1}}(x)=w(x, 0), \quad \Pi_{\gamma_{3}}(x)=\left.\left\{\frac{K_{d}}{2} \partial_{\tilde{\rho}}^{2}+\mathrm{a} \partial_{\tilde{\rho}}\right\} w(x, \rho)\right|_{\rho=0}, \\
& \Pi_{\gamma_{2}}(x)=\left.\partial_{\tilde{\rho}} w(x, \rho)\right|_{\rho=0}, \quad \Pi_{\gamma_{4}}(x)=\left.\left\{-\frac{K_{d}}{3 !} \partial_{\tilde{\rho}}^{3}-\frac{C_{d}}{12} \partial_{\tilde{\rho}}\right\} w(x, \rho)\right|_{\rho=0},
\end{aligned}
$$

with $\left(K_{d}, C_{d}\right)=(5,50),(3,42),(2,44),(1,34)$ for $d=5,6,8,10$, respectively.

Analytic continuation of $\Pi_{\gamma_{4}}(x)$ about the relevant conifold point has a similar form to (2.12). Then the suitable Laplace transforms of $\Pi_{k}(x)$, see (2.13), give the asymptotic series $g_{k}(z)$. For all cases of mirror hypersurfaces 
$Y(d)$, one verifies the relations

$$
K_{R}=M_{\text {Con. }}, \quad K_{\bar{R}} L=M_{0},
$$

which connect the monodromy of $\Pi_{\gamma_{k}}(x)$ to the Stokes matrices of the holomorphic solution $\Phi_{l}(z)$.

\section{Acknowledgments}

C.F.D. would like to thank the Graduate School of Mathematical Sciences, University of Tokyo, and the Perimeter Institute, Waterloo, for supporting his visits during the critical early stages of this project. S.H. would like to thank the organizers of the research programs "Mathematical Structures in String Theory 2005" at KITP, Santa Barbara, and "Modular Form and String Duality" at BIRS, Banff (June 2006), for providing him nice research environments where this work has progressed a lot. We thank A. Corti, J. Morgan, and M.-H. Saito for valuable discussions. C.F.D. is supported in part by a Royalty Research Fund Scholar Award from the Office of Research, University of Washington. S.H. is supported in part by the Grant-in Aid for Scientific Research C18540014.

\section{References}

[1] S. Cecotti and C. Vafa, Ising model and $N=2$ supersymmetric theories, Commun. Math. Phys. 157 (1993), 139-178.

[2] B. Dubrovin, Geometry and integrability of topological-antitopological fusion, Commun. Math. Phys. 152 (1993), 539-564.

[3] S. Cecotti and C. Vafa, On classification of $N=2$ supersymmetric theories, Commun. Math. Phys. 158 (1993), no. 3, 569-644.

[4] K. Hori, A. Iqbal and C. Vafa, D-branes and mirror symmetry, hepth/0005247.

[5] D. Auroux, L. Katzarkov and D. Orlov, Mirror symmetry for weighted projective planes and their noncommutative deformations, math.AG/0404281.

[6] B. Dubrovin, Geometry and analytic theory of Frobenius manifolds, Proceedings of the International Congress of Mathematicians, Vol. II (Berlin, 1998), Doc. Math. 1998, Extra Vol. II, 315-326.

[7] D. Guzzetti, Stokes matrices and monodromy of the quantum cohomology of projective spaces, Comm. Math. Phys. 207 (1999), no. 2, $341-383$. 
[8] P. Candelas, X.C. de la Ossa, P.S. Green and L. Parkes, A pair of Calabi-Yau manifolds as an exactly soluble superconformal theory, Nucl. Phys. B 356 (1991), 21-74.

[9] V.I. Arnold, S.M. Gusein-Zade and A.N. Varchenko, Singularities of differential maps, Vol. II, Birkhäuser, 1988.

[10] K. Saito, Period mapping associated to a primitive form, Publ. RIMS Kyoto Univ. 19 (1983), 1231-1264.

[11] D.R. Morrison, Picard-Fuchs equations and mirror maps for hypersurfaces, in 'Essays on Mirror Manifolds' ed. S.-T. Yau Internal Press, Hong Kong, 1992, 241-264.

[12] A. Klemm and S. Theisen, Considerations of one-modulus CalabiYau compactifications: Picard-Fuchs equations, Kähler potentials and mirror maps, Nucl. Phys. B 389 (1993), no. 1, 153-180.

[13] E.A. Coddington and N. Levinson, Theory of ordinary differential equation, McGraw-Hill, 1995.

[14] S. Hosono, Central charges, symplectic forms, and hypergeometric series in local mirror symmetry, hep-th/0404043.

[15] S. Hosono, Local mirror symmetry and type IIA monodromy of CalabiYau manifolds, Adv. Theor. Math. Phys. 4 (2000), 335-376.

[16] V.V. Batyrev, Variations of the mixed Hodge structure of affine hypersurfaces in algebraic tori, Duke Math. J. 69 (1993), 349-409.

[17] A. Strominger, S.-T. Yau and E. Zaslow Mirror symmetry is T-duality, Nucl. Phys. B 479 (1996), 243-259.

[18] V.V. Batyrev, Dual polyhedra and mirror symmetry for Calabi-Yau hypersurfaces in toric varieties, J. Alg. Geom. 3 (1994), 493-535.

[19] I.M. Gel'fand, A.V. Zelevinski and M.M. Kapranov, Equations of hypergeometric type and toric varieties, Funktsional Anal. i. Prilozhen. 23 (1989), 12-26; Funct. Anal. Appl. 23 (1989), 94-106 (In English).

[20] S. Hosono, A. Klemm, S. Theisen and S.-T. Yau, Mirror symmetry, mirror map and applications to Calabi-Yau hypersurfaces, Commun. Math. Phys. 167 (1995), 301-350.

[21] J. Stienstra, Resonant hypergeometric systems and mirror symmetry, in 'Integrable systems and algebraic geometry (Kobe/Kyoto 1997)', World Scientific Publishing, River Edge, NJ, 1998, 412-452.

[22] C.F. Doran and J.W. Morgan, Mirror symmetry and integral variations of Hodge structure underlying one parameter families of Calabi-Yau threefolds, math.AG/0505272.

[23] C. Enckevort and D. van Straten, Monodromy calculations of fourth order equations of Calabi-Yau type, math.AG/0412539. 
[24] M. Gross, Special lagrangian fibrations. I. topology, Integrable Systems and Algebraic Geometry '(Kobe/Kyoto, 1997)', World Scientific Publishing, River Edge, NJ, 1998, 156-193.

[25] I. Zharkov, Torus fibrations of Calabi-Yau hypersurfaces in toric varieties, Duke Math. J. 101 (2000), no. 2, 237-257.

[26] W.-D. Ruan, Lagrangian torus fibrations and mirror symmetry of Calabi-Yau manifolds, in 'Symplectic geometry and Mirror Symmetry (Seoul, 2000)', World Scientific Publishing, River Edge, NJ, 2001, $385-427$.

[27] I.M. Gel'fand, A.V. Zelevinski and M.M. Kapranov, Discriminants, resultants and multidimensional determinants, Birkhäuser, Boston, 1994.

[28] G. Mikhalkin, Decomposition into pairs-of-pants for complex algebraic hypersurfaces, Topology 43 (2004), no. 5, 1035-1065.

[29] M. Abouzaid, Homogeneous coordinate rings and mirror symmetry for toric varieties, math.SG/0511644.

[30] M. Kontsevich, Homological algebra of mirror symmetry, Proceedings of the International Congress of Mathematicians (Zürich, 1994), Birkhäuser, 1995, $120-139$. 